Post-print of: Microporous and Mesoporous Materials Volume 149, Issue 1, 1 February 2012, Pages 142-146

\title{
Magnetron sputtered a-SiOxNy thin films: A closed porous nanostructure with controlled optical and mechanical properties
}

\author{
V. Godinho, T.C. Rojas, A. Fernández \\ Instituto de Ciencia de Materiales de Sevilla CSIC-US, Av. Américo Vespucio 49, 41092 Sevilla, \\ Spain
}

\begin{abstract}
Amorphous silicon oxynitride coatings with similar composition and different closed porosity were prepared by magnetron sputtering. Pores size, shape and distribution were evaluated by scanning electron microscopy and transmission electron microscopy. Raman and EELS analysis proved that the pores are filled with molecular nitrogen trapped during deposition.

The mechanical properties evaluated by nanoindentation shows that the presence of closed nano-porosity does not compromise the mechanical integrity of these coatings. The introduction of closed porosity is shown as a good strategy for obtaining lower dielectric constant silicon oxynitride coatings with similar composition while keeping the good mechanical properties ( $\sim 13 \mathrm{GPa})$ characteristic of this type of coatings. The presence of close porosity gives also a good stability of coatings properties as compared to open porosity microstructures where gas phase in contact with the coatings can affect coatings properties.
\end{abstract}

\section{Graphical abstract}

Amorphous silicon oxynitride coatings with similar composition and different closed porosity were prepared by magnetron sputtering. EELS analysis revealed that molecular nitrogen is trapped inside the pores during deposition. The introduction of closed porosity is shown as a good strategy for obtaining low dielectric constant silicon oxynitride coatings with similar composition, keeping the good mechanical properties characteristic of this type of coatings.

\section{Keywords}

Silicon oxynitride; Closed porosity; Tailored refractive index; Mechanical stability

\section{Introduction}

Over the last decades amorphous silicon oxynitride coatings have been the subject of great interest not only due to their excellent properties such as chemical inertness, high thermal stability and corrosion resistance, but also due to their combination of adequate mechanical, optical and electrical properties [1], [2], [3], [4] and [5]. Silicon oxynitride coatings have been 
considered as an interesting alternative to silicon oxide coatings with improved properties as diffusion barrier for boron and phosphor [6] and low mechanical stress [1]. The dielectric constant of SiON coatings increases linearly with the amount of nitrogen in the coatings from 3.8 of $\mathrm{SiO} 2$ to 7.8 of Si3N4[7]. A continuous change in its composition allows to obtain films with different optical, electrical and mechanical properties [3], [8] and [9]. The possibility of tuning these properties is important for many industrial applications such as passivation and masking layers in microelectronics, solar cells, luminescent devices [10] and [11] or antireflection coatings [12] and [13].

In an attempt to lower the dielectric constant of several materials, open porosity is being introduced [14]. However the weaker mechanical strength of these new porous dielectric materials is one of the major drawbacks for its integration in microelectronic devices. Additionally, open porosity may allow the diffusion of water and other impurities. In a previous work we reported for the first time the introduction of closed porosity in silicon oxynitride coatings [15] deposited by magnetron sputtering by changing the composition of the gas phase. We showed how this nano-porosity allows to have silicon oxynitride coatings with similar chemical and mechanical properties but with different refractive index when compared to non-porous coatings. The present paper is complementing these previous results by studying the formation of porous silicon oxynitride coatings by controlling the geometrical parameters in addition to gas phase composition during magnetron sputtering deposition to obtain this singular closed porous nanostructure. The paper includes an exhaustive microstructural analysis in the nanoscale by transmission electron microscopy (TEM) with high angle annular dark filed (HAADF) detection, electron energy-loss spectroscopy (EELS) and Raman spectroscopy as well as an evaluation of the influence of the nanostructure on the optical and mechanical properties.

\section{Experimental}

Silicon oxynitride coatings with closed porosity were deposited according to Refs. [15] and [16] on $\mathrm{Si}\left(\begin{array}{ll}1 & 0\end{array}\right)$, steel and glass substrates by reactive magnetron sputtering from a pure $\mathrm{Si}$ target (Kurt J. Lesker 99.999\%), using an RF sputtering source at a power of $150 \mathrm{~W}$. Before deposition, the base pressure was $8 \times 10-5 \mathrm{~Pa}$. A mixture of nitrogen and oxygen (total pressure of 1.33 $\mathrm{Pa}$, with a partial pressure of oxygen of $1 \times 10-4 \mathrm{~Pa}$ ) was used as sputtering gas. The target to substrate distance was 5 or $10 \mathrm{~cm}$. These samples are hereafter referred to as \$150-5 and S150-10, respectively.

Also a dense silicon oxynitride coating was deposited for comparison. In this case and to avoid any type of porosity the coating was deposited at $300 \mathrm{~W} \mathrm{rf}$ with a dc bias of $500 \mathrm{~V}$, keeping a target-substrate distance of $10 \mathrm{~cm}$ (sample S300). Table 1 resumes the deposition conditions.

The thickness and morphology of the samples were studied by scanning electron microscopy (HITACHI S-4800 SEM-FEG). The samples were cleaved from coatings grown onto silicon, and were observed without metallization in cross-sectional views at 1-2 kV. 
The composition of the films was determined by X-ray photoelectron spectroscopy (XPS). A Leybolb Heraeus LH10 Spectrometer was used, working in the constant analyzer energy mode with a pass-energy of $50 \mathrm{eV}$. The binding energy reference was taken as the main component of the C1s peak at $284.6 \mathrm{eV}$ for adventitious carbon. For quantification the XPS spectra were subjected to background subtraction (Shirley background) and sensitivity factors supplied by the instrument manufacturer were used.

The microstructure of the coatings, pore size and distribution, was investigated using a TEM Philips CM200 microscope. Cross sectional TEM specimens were prepared in the conventional way by mechanical polishing followed by $\mathrm{Ar}+$ ion milling to electron transparency.

High Angle Annular Dark Field (HAADF), Scanning Transmission Electron Microscopy (STEM) images and electron energy-loss spectra (EELS) were acquired on a JEOL 2010 field emission gun transmission electron microscope operated at $200 \mathrm{kV}$. The spectrum imaging mode, consisting in acquiring a series of EELS spectra while a $0.5 \mathrm{~nm}$ beam with a current of 0.1-0.3 nA scanned along the sample area, was used. The HAADF signal was also simultaneously collected at each point within the scanned area. This approach allows correlating nanoanalytical and structural information from the dense part of the coating and from the pores.

Raman spectra measurements were carried out with a LabRAM Horiba Jobin Yvon spectrometer equipped with a charge-coupled device detector and a He-Ne laser (532 nm) at $5 \mathrm{~mW}$. Since the Raman signal of steel substrates is weaker than the one from silicon substrates the Raman spectra were measured from samples grown onto steel.

Optical characterization was performed on films deposited on glass and silicon substrates, using a Fourier transform vis-IR spectrophotometer (Bruker IFS-66) attached to a microscope, operating in reflection mode. A $4 \times$ objective with a numerical aperture of 0.1 (light cone of $\pm 5.7^{\circ}$ ) was used to irradiate the sample and collect the reflected light at quasi-normal incidence. The refractive index of the silicon oxynitride coatings was determined by fitting the optical specular reflectance using spectra generated by a Matlab code based on scalar wave approximation, as described elsewhere [17].

The mechanical properties (hardness and reduced Young modulus) of the samples were obtained by nanoindentation, using a Hysitron Inc. with a Berkovich tip at a maximum load of $1000 \mu \mathrm{N}$. The Berkovitch tip area function was estimated with the Oliver-Pharr method [18]. Indentations were made within a depth of approximately $1 / 10$ of the thinner films thickness so that the values of hardness and reduced Young modulus could be measured without the influence of the substrate.

\section{Results and discussion}

Table 2 presents the chemical composition of the samples as given by XPS. The coatings are mainly composed by $\mathrm{Si}, \mathrm{N}$ and $\mathrm{O}$ presenting similar composition. A low carbon signal could be detected due to post deposition contamination by air exposure or handling. Also the Auger parameter of silicon (binding energy of Si2p photoelectron peak plus the kinetic energy of Si KLL Auger peak) was evaluated and is presented in Table 2. The obtained values for samples 
before $(\alpha 1)$ and after argon bombardment $(\alpha 2)$ for surface cleaning are characteristic of silicon oxynitride [19]. Although the partial pressure of oxygen in the gas phase is very low, the high reactivity of silicon with oxygen leads to the formation of silicon oxynitride coatings with reproducible composition as determined by XPS.

The microstructural information as given by SEM-FEG is presented in Fig. 1. As described in our previous work [15] and [16], the deposition of SiON coatings with relatively high partial pressure of reactive gas and under low sputtering power originates coatings with closed porosity containing molecular nitrogen. As can be seen in Fig. 1 sample S150-5 deposited at 5 $\mathrm{cm}$ presents smaller pore size and pore density as compared to sample S150-10 deposited at $10 \mathrm{~cm}$. The control sample, deposited with substrate bias is completely dense. These results show that under low power conditions (and without substrate bias), changing the target to substrate distance allows to control the pore size, provided that pressure and composition of the gas phase is controlled.

The differences in pore size are more evident in the TEM cross-sectional micrographs of Fig. 2, in which it is easier to measure the pore size. At $5 \mathrm{~cm}$ target-substrate distance, the average pore diameter is around $5 \mathrm{~nm}$ while at $10 \mathrm{~cm}$ distance the pore sizes range from 3 to $20 \mathrm{~nm}$.

On our previous work [15] we showed evidence by Raman spectroscopy that the pores are closed and filled with molecular nitrogen trapped during deposition. To confirm these findings, Raman spectra of both porous samples were measured and are presented in Fig. 3 as compared to the fully dense $S 300$ sample. The band at around $2330 \mathrm{~cm}-1$ corresponds to molecular nitrogen [15] and [20]. The lower intensity of the Raman signal for the sample prepared at $5 \mathrm{~cm}$ with smaller pore size is an indication of lower amount of molecular nitrogen incorporated.

EELS analysis in the nanoscale is a powerful technique that allows to have information on the bonding state of the elements in the coatings with a lateral resolution of about $0.5 \mathrm{~nm}$. Combined with HAADF/STEM, where the signal intensity is proportional to the atomic number and the thickness of the sample $(\mathrm{I} \propto \mathrm{CZ} 2 \mathrm{Nt}$, where $\mathrm{I}$ is the intensity, $\mathrm{C}$ a constant, $\mathrm{Z}$ the atomic number and $t$ the thickness), the presence of a different phase and/or voids can be clearly identified. These techniques with high lateral resolution in the nanoscale were employed to study sample S150-10.

The HAADF/STEM image on Fig. 4a reveals the highly porous structure of sample S150-10, where the dark areas correspond to phases with low $Z$, in this case the pores. The decay of intensity of the HAADF profile across the marked line clearly evidences when a pore is reached.

The fine structure of the core edges is known to be sensitive to the local atomic environment. Taking advantage of the high nanoscale lateral resolution in the STEM-FEG microscope, EELS analysis of the $\mathrm{N}-\mathrm{K}$-edge can be performed inside and outside the pores to investigate the bonding state of nitrogen (see Fig. $4 \mathrm{~b}$ ). Also the O-K and Si-L2,3 edges, have been measured inside and outside the pores. In Fig. $4 \mathrm{~b}$, the $\mathrm{N}$ and $\mathrm{O}-\mathrm{K}$ and Si-L2,3 edges, inside and outside of the marked pore are presented. Outside the pore (position 10) the N K-edge corresponds to $\mathrm{N}$ in SiON, while the N K-edge inside the pore (position 30) is similar to molecular nitrogen [21] and [14]. In the last case also some contribution in the spectrum from nitrogen forming the 
SiON phase cannot be neglected, due to the layer surrounding the pore. These data confirm the Raman results, the pores are filled with molecular nitrogen trapped during deposition. The Si-L2,3 ionization edge in Fig. 4b is typical of a silicon oxynitride coating [15] and [22] and no changes are observed from both areas. The Si signal measured from inside the pore is due to the pore walls. Proving in conclusion, the presence of closed porosity filled with molecular nitrogen in a matrix of amorphous silicon oxynitride.

To evaluate the effects of the different porosity on the optical properties of the SiON coatings, vis-IR spectra were measured in the range 450-1200 $\mathrm{nm}$. The thickness and refractive index were obtained trough simulation of these spectra using the scalar wave approximation [17] and the best fits are presented in Table 3.

As expected the coatings present refractive index values between those of silicon oxide (1.47) and silicon nitride (2.3) in the visible range and the porous samples (S150-10 and S150-5) present lower $\mathrm{n}$ when compared to the dense control sample. The dielectric constant derived from the measurements of refraction index in the vis-IR region for sample $S 300$ is also between the published values for SiO2 (2.138) and Si3N4 (4.89) [23] at these high frequencies. The porous coatings with similar composition denote the influence of porosity in decreasing the dielectric constant and how the deposition method employed is effective to change the properties of these coatings.

If we take effective medium approximations, expressing the relative electrical permittivity of an heterogeneous media ( $\varepsilon r$,eff) according to Maxwell-Garnett theory, as a function of the dielectric constants of the continuous $(\varepsilon r, c)$ and dispersed phase $(\varepsilon r, d)$ it is possible to calculate the volume fraction $(\phi)$ occupied by the dispersed phase, the pores. Considering the dielectric constant of sample $\mathrm{S} 300$ as being correspondent to the continuous phase, the volume pore fraction of samples S150-10 and S150-5 can be calculated (Table 3). Sample S15010 presents a pore volume fraction of $26 \%$ while sample S150-5 has $17 \%$ pore volume, which can explain the differences in the refractive index. An introduction of $17 \%$ porosity causes a substantial decrease of the refractive index from 1.87 to 1.74 . Increasing the target-substrate distance increases the pore volume fraction to $26 \%$ and an even a lower refractive index of 1.60 is achieved. The pore density as observed by XTEM in Fig. 2 is in fact a superposition of pores along the full XTEM sample thickness (around $100 \mathrm{~nm}$ ).

One of the most interesting expected features of having coatings with different dielectric constant and closed porosity is their mechanical stability. The mechanical properties of these samples were evaluated by nanoindentation. Table 4 presents the results of hardness and reduced Young modulus comparing the dense SiON coating and the porous samples. No appreciable changes are observed and the coatings present very high hardness and elastic modulus when compared to other materials with low dielectric constant and similar pore fraction [14] and [24], making of these porous silicon oxynitride coatings obtained by magnetron sputtering, good candidates for applications that require high mechanical strength and structural integrity.

\section{Conclusions}


In this work the preparation of amorphous silicon oxynitride coatings showing a closed porous nanostructure with similar composition has been achieved by using the magnetron sputtering methodology. Different target to substrate distances can be used to control not only pore size but also the volume fraction of pores introduced in the coatings and so a substantial decrease of the refractive index and dielectric constant have been measured as compared to a fully dense reference material.

The EELS and Raman results proved that the closed pores are filled with molecular nitrogen. This structure of closed porosity allows not only to have thin films with different dielectric constant but also to keep the good mechanical properties typical of silicon oxynitride coatings. The presence of close porosity gives also a good stability of coatings properties as compared to open porosity microstructures in regards to the influence of environment gases. Their outstanding mechanical properties when compared to coatings with similar dielectric constant, allied with the easiness of the deposition method to be scaled up to industrial processes, makes these coatings very interesting for different applications.

Future works are still to be done to elucidate the mechanism of formation of this closed porosity during deposition. Nevertheless the potentiality of the magnetron sputtering methodology has been demonstrated in this work.

\section{Acknowledgments}

Authors thank the financial support from EC (NANOINDENT and REGPOT AL-NANOFUNC), CSIC (201060I041 and 201060E102), Spainish Ministry MICINN (Consolider FUNCOAT) and Junta de Andalucia (TEP 217). 


\section{References}

[1] D. Criado, M.I. Alayo, M.C.A. Fantini, I. Pereyra

Journal of Non-Crystalline Solids, 352 (2006), pp. 2319-2323

[2] M.L. Green, E.P. Gusev, R. Degraeve, E.L. Garfunkel

Journal of Applied Physics, 90 (2001), pp. 2057-2121

[3] L. Martinu, D. Poitras

Journal of Vacuum Science \& Technology, A: Vacuum, Surfaces, and Films, 18 (2000), pp. 26192645

[4] M. Vetter

Thin Solid Films, 337 (1999), pp. 118-122

[5] M. Vogt, R. Hauptmann

Surface Coatings and Technology, 74-75 (1995), pp. 676-681

[6] P. Hoffmann, D. Schmeißer, R.B. Beck, M. Cuch, M. Giedz, A. Jakubowski

Journal of Alloys and Compounds, 382 (2004), pp. 228-233

[7] D.M. Brown, P.V. Gray, F.K. Heumann, H.R. Philipp, E.A. Taft

Journal of the Electrochemical Society, 115 (1968), p. 311

[8] B.K. Yen, R.L. White, R.J. Waltman, Q. Dai, D.C. Miller, A.J. Kellock, B. Marchon, P.H. Kasai, M.F. Toney, B.R. York, H. Deng, Q.-F. Xiao, V. Raman

Journal of Vacuum Science \& Technology, A: Vacuum, Surfaces, and Films, 21 (2003), pp. 18951904

[9] M.I. Alayo, D. Criado, L.C.D. Gonçalves, I. Pereyra

Journal of Non-Crystalline Solids, 338-340 (2004), pp. 76-80

[10] T.S. Eriksson, C.G. Granqvist

Journal of Applied Physics, 60 (1986), pp. 2081-2091

[11] M. Ribeyro, I. Pereyra, M.I. Alayo

Thin Solid Films, 426 (2003), pp. 200-204

[12] M. Serényi, M. Rácz, T. Lohner

Vacuum, 61 (2001), pp. 245-249

[13] E.C. Samano, J. Camacho, R. Machorro 
Journal of Vacuum Science \& Technology, A: Vacuum, Surfaces, and Films, 23 (2005), pp. 12281233

[14] M.R.B.K. Maex, D. Sharmiryan, F. lacopi, S.H. Brongersma, Z.S. Yanovitskaya

Journal of Applied Physics, 93 (2003), pp. 8793-8841

[15] V. Godinho, M.C.J. de Haro, J. Garcia-Lopez, V. Goossens, H. Terryn, M.P. DelplanckeOgletree, A. Fernandez

Applied Surface Science, 256 (2010), pp. 4548-4553

[16] V. Godinho, A. Fernandez, Procedimiento de obtención de recubrimientos mediante pulverización catódica y recubrimiento obtenible mediante dicho procedimiento, Spain, P200930085, PCT/ES 2010/070245 (for more details please contact asuncion@icmse.csic.es).

[17] D.M. Mittleman, J.F. Bertone, P. Jiang, K.S. Hwang, V.L. Colvin

Journal of Chemical Physics, 111 (1999), pp. 345-354

[18] W.C. Oliver, G.M. Pharr

Journal of Materials Research, 7 (1992), pp. 1564-1583

[19] D. Briggs, M.P. Seah

Practical Surface Analysis (1990)

[20] V. Godinho, V.N. Denisov, B.N. Mavrin, N.N. Novikova, E.A. Vinogradov, V.A. Yakovlev, C. Fernádez-Ramos, M.C.J.d. Haro, A. Fernández

Applied Surface Science, 256 (2009), pp. 156-164

[21] C.C. Ahn, O.L. Krivanek

EELS Atlas

Gatan Inc. (1983)

[22] L. Torrison, J.K.J. Tolle, S.K. Dey, D. Gu, I.S.T. Tsong, P.A. Crozier

Materials Science \& Engineering, B: Solid-State Materials for Advanced Technology, 97 (2003), pp. $54-58$

[23] X. Tan, J. Wojcik, P. Masher

Journal of Vacum Science and Technology A, 22 (4) (2004), pp. 1115-1119

[24] X. Li, X. Yin, L. Zhang, L. Cheng, Y. Qi

Materials Science \& Engineering, A: Structural Materials: Properties, Microstructure and Processing, 500 (2009), pp. 63-69 


\section{Figure captions}

Figure 1. SEM cross-sectional views: (a) sample S150-5, (b) sample S150-10 and (c) sample S300.

Figure 2. XTEM micrographs of the porous coatings grown at different target-substrate distance: (a) sample S150-5 and (b) sample S150-10.

Figure 3. Raman spectra of samples S150-10, S150-5 and S300.

Figure 4a. HAADF/STEM image of sample S150-10 and HAADF profile across a pore (marked line).

Figure $4 \mathrm{~b}$. EELS spectra of $\mathrm{N}$ and $\mathrm{O}$ K-edge and Si-L2,3-edge outside and inside the pore (corresponding to 10 and 30 position in the HAADF profile). 
Table 1. Deposition conditions.

\begin{tabular}{|l|l|l||l|l|l||}
\hline Sample & $\begin{array}{l}\text { Rf power } \\
\text { W) }\end{array}$ & $\begin{array}{l}\text { Sub. } \\
\text { Bias } \\
(\mathbf{V})\end{array}$ & $\begin{array}{l}\text { Target-substrate } \\
\text { distance }(\mathbf{c m})\end{array}$ & $\begin{array}{l}\text { Thickness } \\
(\mathbf{n m})\end{array}$ & $\begin{array}{l}\text { Deposition rate } \\
(\mathbf{n m} / \mathbf{m i n})\end{array}$ \\
\hline $\begin{array}{l}\text { S150- } \\
10\end{array}$ & 150 & - & 10 & 400 & 2.2 \\
\hline S150-5 & 150 & - & 5 & 2500 & 14 \\
\hline S300 & 300 & 500 & 10 & 530 & 2.9 \\
\hline
\end{tabular}


Table 2. Chemical composition as given by XPS.

\begin{tabular}{|l|l|l|l||l|l|l|}
\hline Sample & \multicolumn{3}{l|l|}{ Chemical composition (at\%) } & \multicolumn{2}{l|}{ Auger Parameter (eV) } \\
\hline \hline & Si & N & O & C & $\boldsymbol{\alpha}^{\mathbf{1}}$ & $\boldsymbol{\alpha}^{\mathbf{2}}$ \\
\hline S150-10 & 37 & 32 & 26 & 5 & 1713.9 & 1713.8 \\
\hline S150-5 & 36 & 42 & 15 & 7 & 1714.1 & 1714.0 \\
\hline S300 & 38 & 41 & 17 & 4 & 1714.2 & 1714.1 \\
\hline
\end{tabular}


Table 3

Table 3. Refractive index, thickness and dielectric constant of the coatings obtained by simulation of vis-IR spectra. Pore volume fraction given by Maxwell-Garnett equation.

\begin{tabular}{|l|l|l|l|l||}
\hline Sample & $\boldsymbol{n}$ & Thickness (nm) & $\boldsymbol{\varepsilon}$ & $\boldsymbol{\varphi ( \% )}$ \\
\hline $\begin{array}{l}\text { S150- } \\
10\end{array}$ & 1.60 & 425 & 2.77 & 26 \\
\hline S150-5 & 1.74 & 2500 & 3.02 & 17 \\
\hline S300 & 1.87 & 510 & 3.52 & - \\
\hline
\end{tabular}


Table 4. Mechanical properties of the coatings measured by nanoindentation.

\begin{tabular}{|l|l|l|}
\hline Sample & H (GPa) & E (GPa) \\
\hline S150-10 & $12.7 \pm 0.9$ & $126.9 \pm 4.8$ \\
\hline S150-5 & $12.5 \pm 0.7$ & $129.5 \pm 5.5$ \\
\hline S300 & $13.2 \pm 1.2$ & $129.2 \pm 4.5$ \\
\hline
\end{tabular}


Figure 1
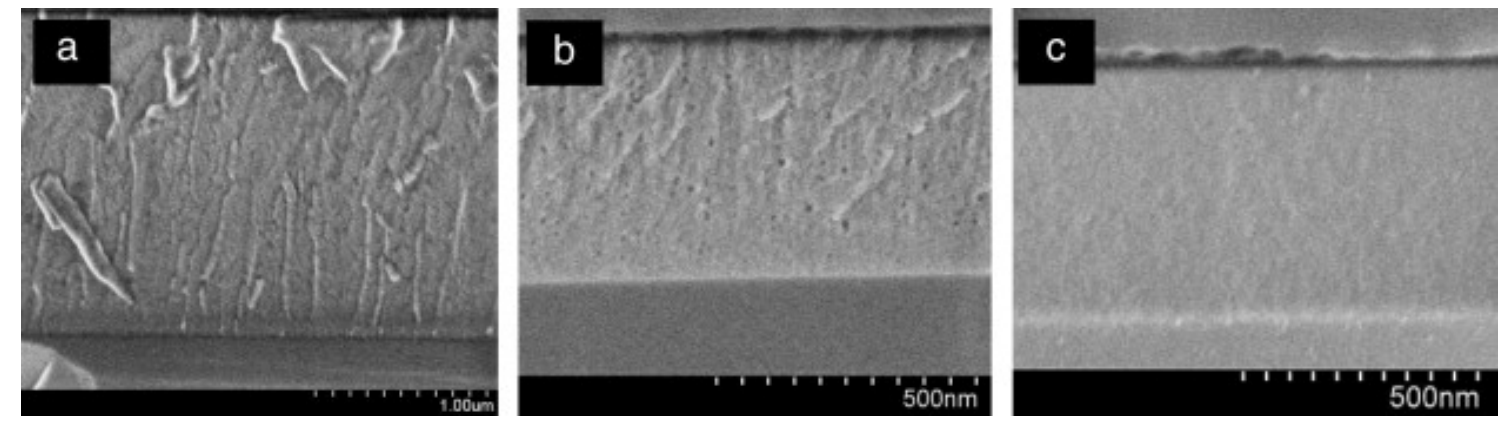
Figure 2

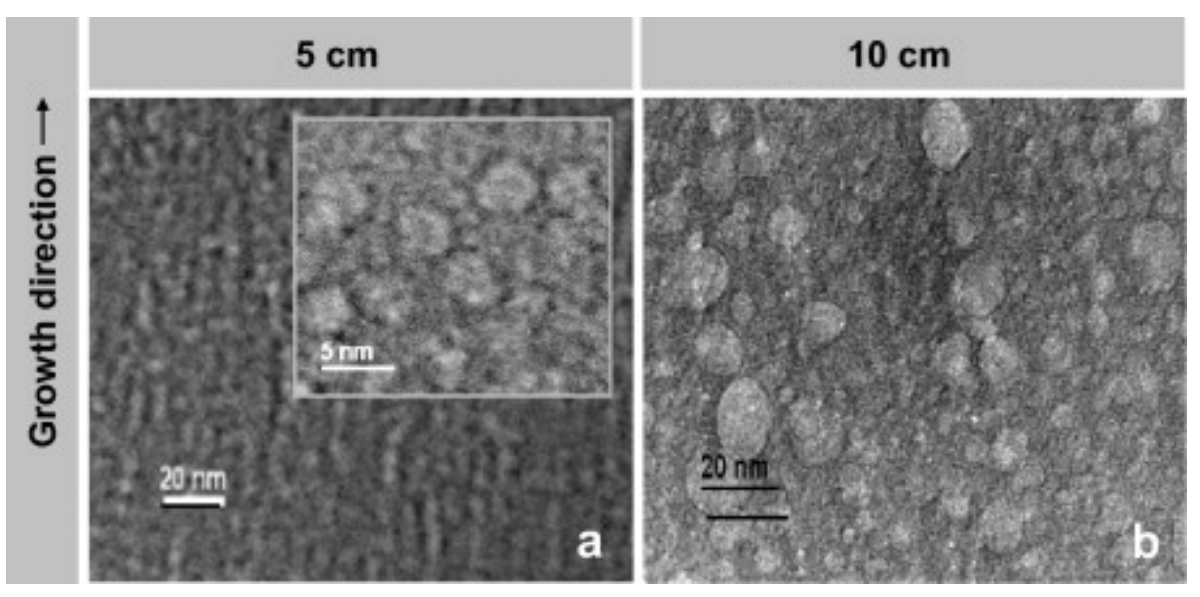


Figure 3

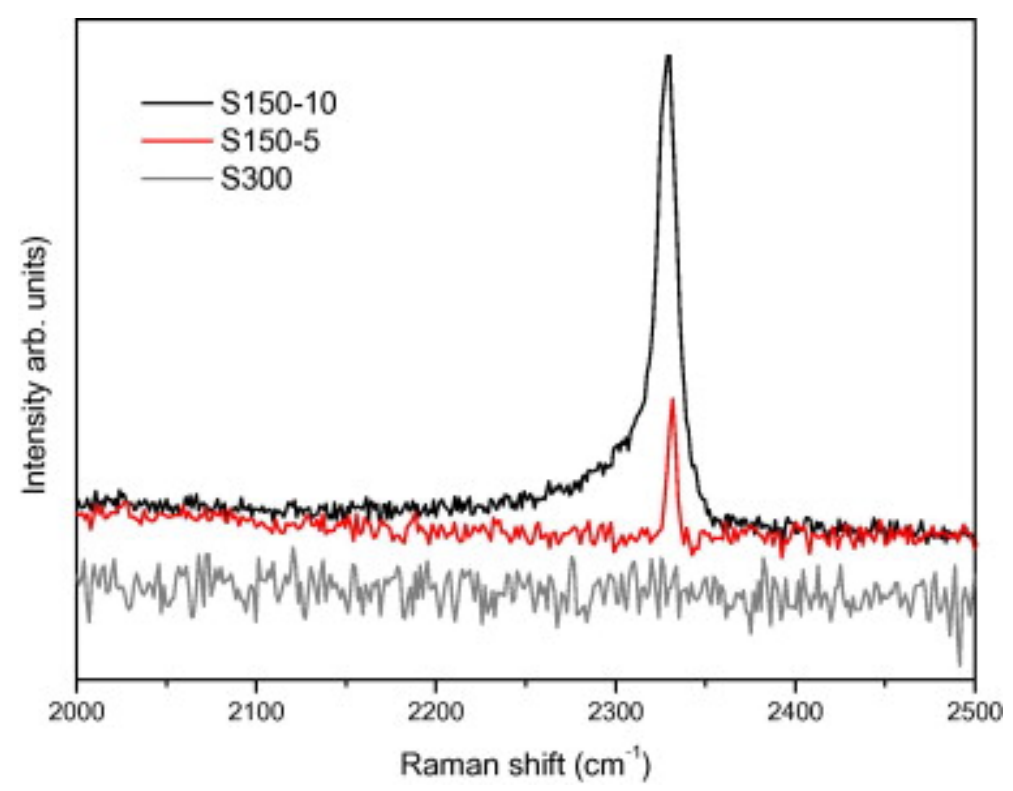


Figure 4a
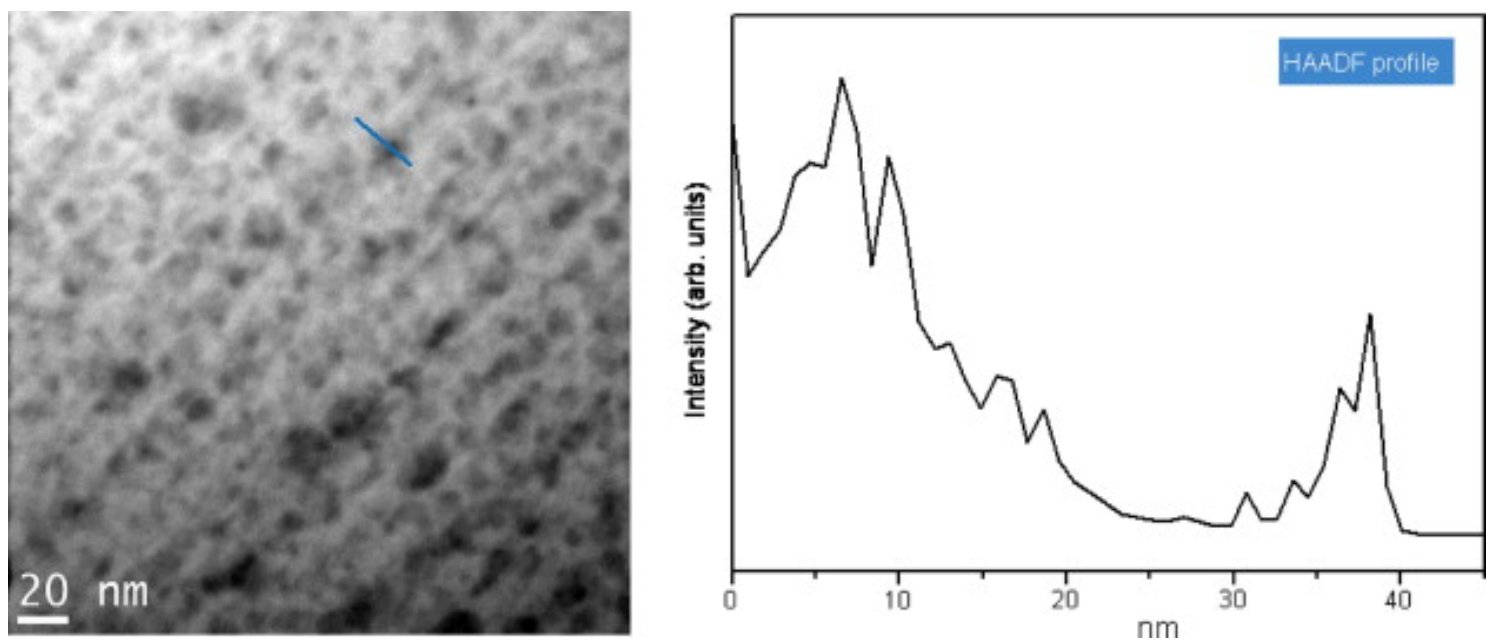

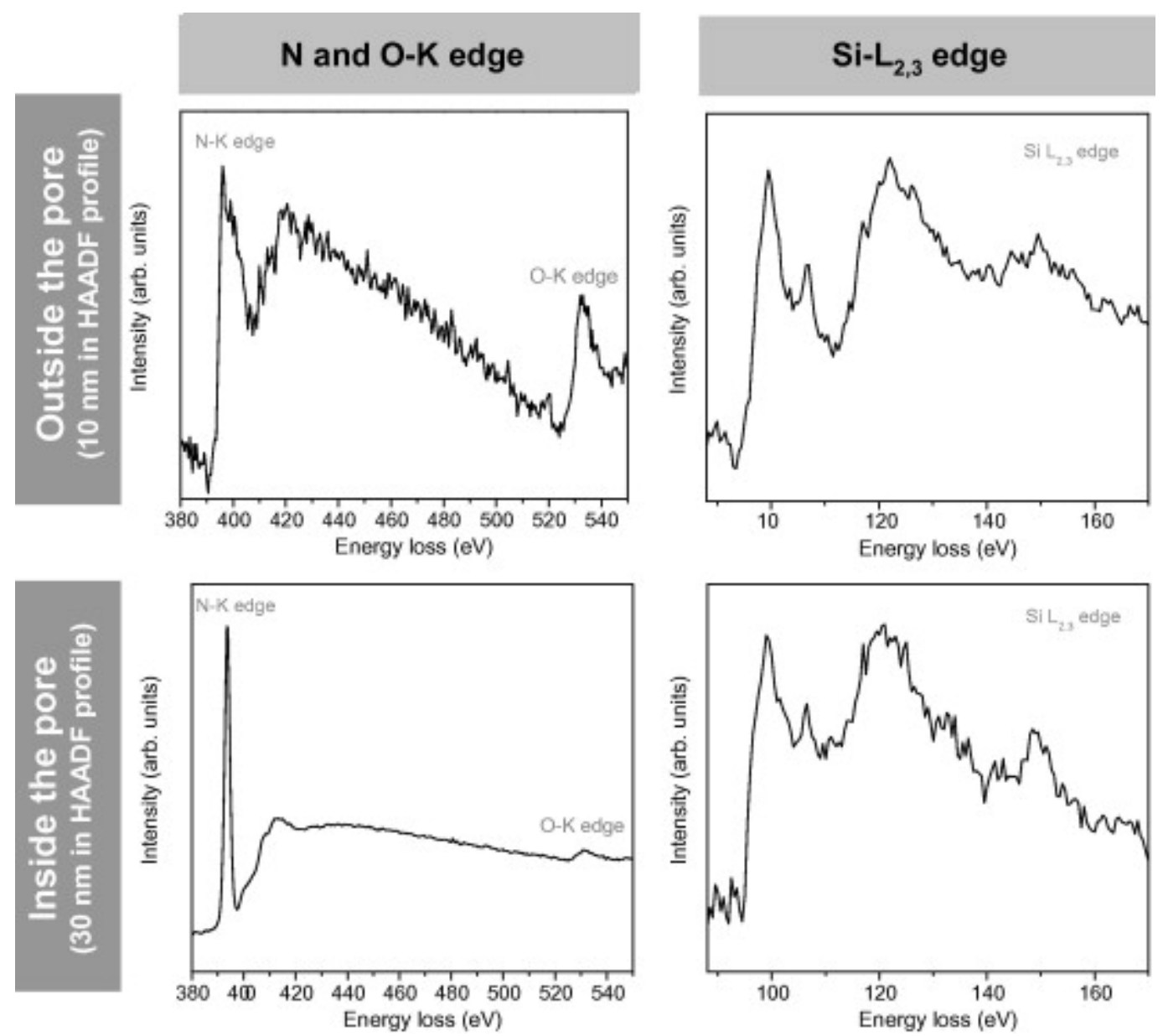